\title{
Der Kniegelenkersatz bei der posttraumatischen Gonarthrose - Standortbestimmung
}

\author{
Dietrich Schulte-Bockholt, Klaus Wendl, Alfred Schmidgen, Andreas Wentzensen
}

\section{Zusammenfassung}

Als Folge einer Fraktur im Bereich des Kniegelenks und sekundär als Folge einer traumatischen Achsfehlstellung kommt es fast regelhaft $\mathrm{zu}$ einer mehr oder minder ausgeprägten Kniegelenksarthrose. Eine fortgeschrittene Gonarthrose lässt sich grundlegend nur durch eine Arthrodese oder einen Kunstgelenkersatz therapieren. Geschildert werden die verschiedenen Prothesentypen, die jeweilige Indikation und die Charakteristika der einzelnen Modelle mit ihren Revisionsraten.

\section{Einleitung}

Weltweit werden immer mehr Knieprothesen bei primärer Gonarthrose implantiert. In Deutschland etwa jährlich 55000 mit einer jährlichen Zuwachsrate von $8 \%$ (Depuy/Deutschland, Persönliche Mitteilungen). In den USA ist die Zahl der implantierten Knieprothesen von 1975 bis 1990 von 10000 auf 150000 angestiegen [5]. Die Tendenz ist weiter zunehmend. Die Erfahrungen scheinen im Großen und Ganzen gut zu sein, obwohl vergleichende kontrollierte Studien nur in geringer Zahl existieren [5].

Im Gegensatz hierzu gibt es bei der Durchschau der Literatur nur wenige Studien, welche die Indikation der posttraumatischen Gonarthrose einbeziehen. Die meisten Veröffentlichungen beschränken sich auf Fallberichte oder zeigen eher mäßige Ergebnisse bei kleinen Fallzahlen in retrospektiven Studien.

OP-JOURNAL 2003; 19: 226-229

(c) Georg Thieme Verlag Stuttgart . New York
Gründe für das deutlich schlechtere Abschneiden der KTP bei sekundärer Gonarthrose sind die schlechteren Ausgangsbedingungen. In der Regel finden wir einen nicht mehr intakten Weichteilmantel, schlechte knöcherne Verhältnisse und eine ligamentäre Imbalance aufgrund des Traumas. Die Infektionsraten bei voroperierten Knien sind deutlich höher [9,10], gleichzeitig ist das Durchschnittsalter der Patienten niedriger, woraus eine höhere Belastung im Alltag resultiert. Dies zieht eine erhöhte Auslockerungsrate nach sich.

Nichtsdestotrotz steigt auch bei der posttraumatischen Gonarthrose die Rate der KTP-Implantationen. Dies jedoch nicht aufgrund der gleich guten Ergebnisse, sondern weil der Patient es fordert.

Bei dieser Diskussion darf jedoch nicht vergessen werden, dass bei erfolgreicher Implantation einer Knietotalendoprothese der Patient, bezüglich der Lebensqualität und des Funktionserhaltes, deutliche Vorteile genießt im Vergleich zur Ar-

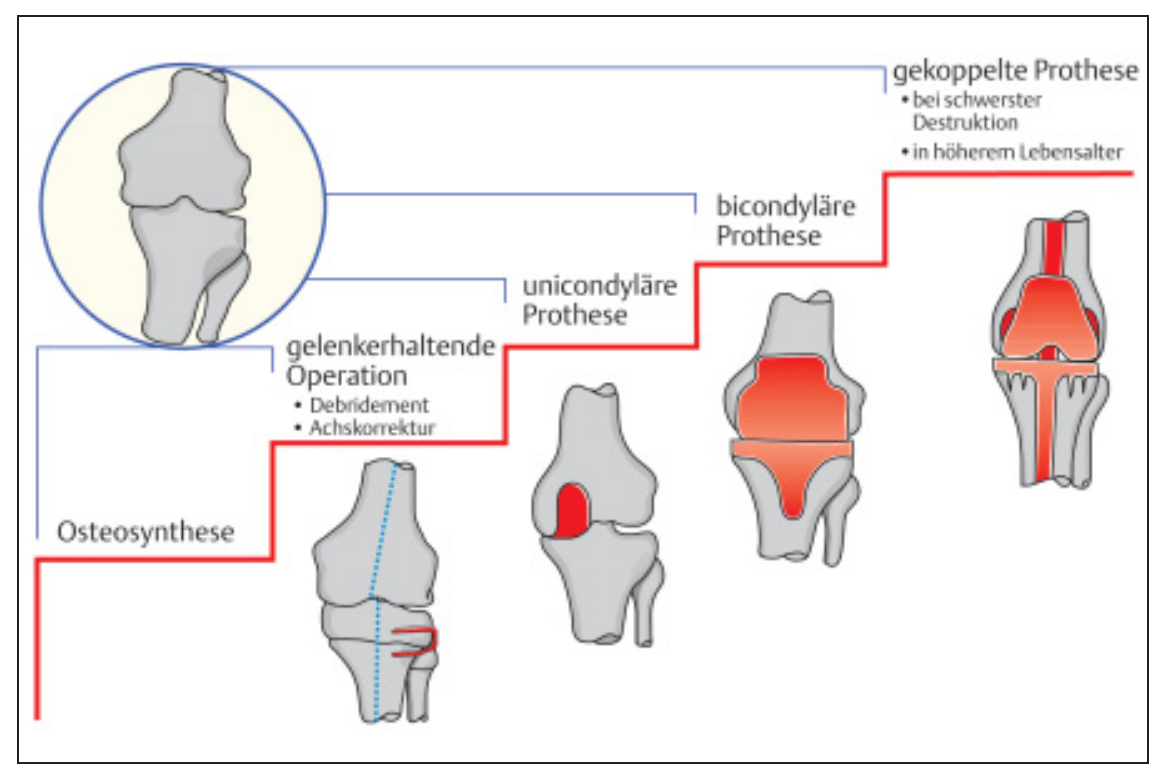

Abb.1 Algorithmus posttraumatische Gonarthrose). throdese des Kniegelenkes, zumindest

Ziel muss es daher sein, den Patienten umfassend zu beraten um den richtigen Prothesentyp und den richtigen Zeitpunkt zu finden und schon bei der Implantation an die erste Revision zu denken.

Eine primäre KTP beim frischen Trauma, wie manche Autoren sie durchführen [7] erscheint unter dem Ziel des Knochenerhalt und der Wiederherstellung der Beinachse mit gutem ligament balancing nicht sinnvoll.

Vielmehr ist, falls die knöchernen und ligamentären Voraussetzungen bestehen, ein Vorgehen in Etappen anzustreben (Abb.1).

Prinzipiell unterscheidet man den unikompartimentellen (Unikondylärer Schlitten) von dem bi-, trikopartimentellen Gelenkersatz (KTP mit oder ohne Patellarückflächenersatz). solange diese nicht ausgelockert ist. 


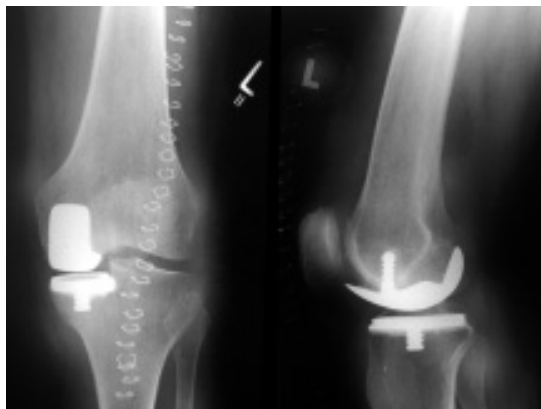

Abb.2 Schlittenprothese.

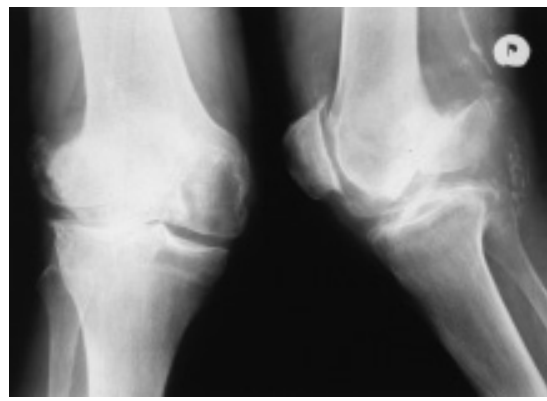

Abb. 3 Posttraumatische Gonarthrose nach Hoffa-Fraktur 1988.

Die Knietotalendoprothese kann dann in gekoppelte und ungekoppelte KTPs unterschieden werden. Dazwischen ist die geführte KTP angesiedelt mit verschiedenen Freiheitsgraden (restrained, semirestrained). Die meisten Prothesen werden zementiert oder zementfrei angeboten, wobei ein „golden standard“, wie bei der Hüfttotalendoprothese, nicht besteht [5].

Die Wahl der geeigneten Prothesen richtet sich primär nach dem Ausmaß der noch bestehenden Bandführung und der Knochenqualität. Des Weiteren müssen Alter, Gewicht und die muskulären Verhältnisse ins Kalkül gezogen werden.

\section{Die Unicondyläre Schlittenprothese}

Die Indikation für eine Unicondyläre Schlittenprothese ist sehr begrenzt. Voraussetzung sind intakte Kreuzbänder und eine Begrenzung des Schadens auf lediglich ein Kompartiment, wie z.B. nach medialer oder lateraler Tibiakopffraktur. Dabei sind die Ergebnisse nach medialer Schlittenimplantation besser als nach lateraler. Eine leichte Seitenbandlaxizität durch ein eingesunkenes Tibiaplateau kann durch ein geeignetes Implantat ausgeglichen werden. Die Beinachse kann nur in geringem Maß korrigiert werden.

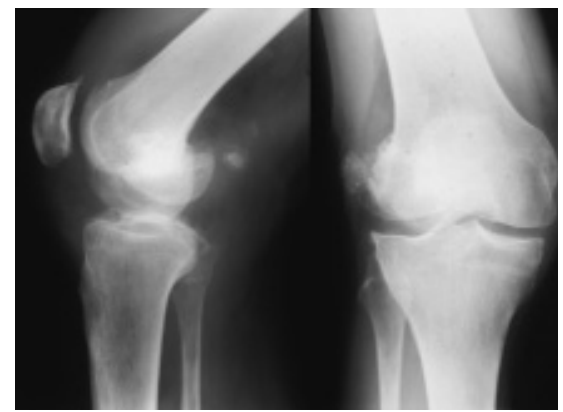

Abb. 4 Präoperativer Zustand.

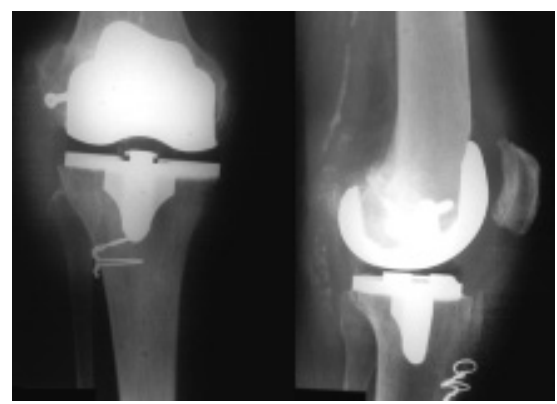

Abb.5 Zustand nach Implantation einer bicondylären Oberflächenprothese mit Osteosynthese der veralteten Hoffa-Fraktur.

Kontraindikationen für eine Unicondyläre Schlittenprothese bilden die Pangonarthrose, die Femoropatellararthrose, größere Abweichung der Beinachse, größere Extensionsdefizite, sowie multidirektionale Instabilitäten (Abb. $\mathbf{2}$ ).

Die Revisionsrate für Unicondyläre Schlitten liegt mit $15 \%$ nach 10 Jahren etwa doppelt so hoch wie bei den Tricompartimentellen Prothesen mit im Schnitt 7,5\% [5].

Die Unicondyläre Schlittenprothese erlebt jedoch momentan eine Renaissance aufgrund verbesserter Ergebnisse durch minimalinvasive Implantation, CAS Navigation und die Anwendung von Mobile Bearing Implantaten [3].

\section{Die Bicondyläre Knietotalendoprothese}

Bei dem größten Teil der momentan implantierten Knietotalendoprothesen handelt es sich um ungekoppelte Oberflächenersatzprothesen (Fallbeispiel Abb. 3-5). Bei noch vorhandenem hinteren Kreuzband und weitestgehend intakter Seitenbandführung ist die Stabilität bei ausgewogenem Ligament Balancing ausreichend, um eine gute Kraftübertragung zu erreichen. Klinische Achsenfehlstellungen bis $30^{\circ}$ können durch Anpassung des Knochenzuschnitts und Auswahl des

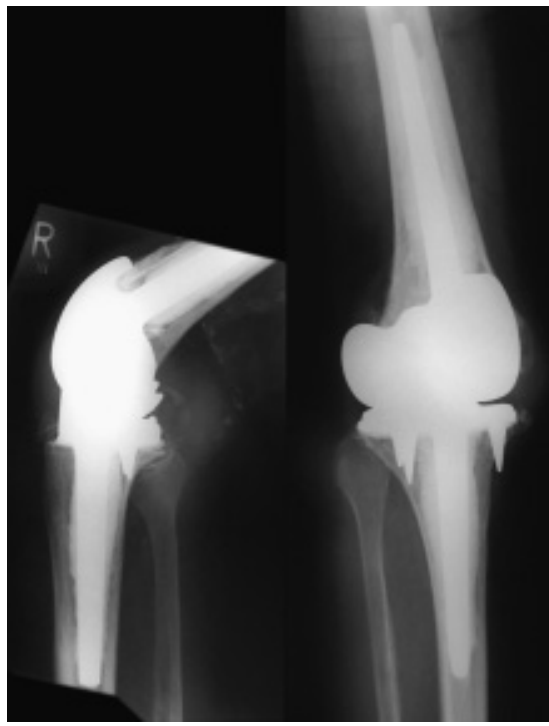

Abb. 6 Gekoppelte Knieprothese Typ Blauth

passenden Implantats ausgeglichen werden. Je nach Ausprägung einer vorhandenen Bandinstabilität, z.B. beim Verlust des vorderen und hinteren Kreuzbandes, kann die Stabilisierung des Gelenkes durch ein spezielles Inlay erreicht werden, das die Bewegung des Knies für die Drehung limitiert.

Kontraindikationen für einen Bicondylären Kniegelenksersatz sind extreme Fehlstellungen der Beinachse, hochgradige Seitenbandinstabilitäten oder auch eine fehlende muskuläre Stabilisierung des Kniegelenks.

Ein Patellarückflächenersatz kann, muss aber nicht zwingend durchgeführt werden. Wichtig ist es aber, eine korrekte Höhe und ein mittiges Gleiten der Kniescheibe zu erreichen.

\section{Die gekoppelte Knietotalendoprothese}

Die gekoppelte Knietotalendoprothese (Abb. 6) kommt dann zum Einsatz, wenn durch körpereigene Mechanismen keine befriedigende Stabilisierung des Kniegelenks gegeben ist. Ausgeprägte Achsfehlstellungen, Pangonarthrosen mit nicht kompensierbarer Seitenbandinstabilität und Revisionseingriffe nach Oberflächenersatz mit großen Knochendefekten und fehlender Bandführung können durch den starren Mechanismus kompensiert werden. Es wird eine primäre stabile Lastübertragung ohne Weichteilbalancing erreicht bei größerer femorotibialer Kongruenz. Jedoch reduziert die achsgeführte Prothese die Freiheitsgrade des Knies auf eine reine Beuge-/ 


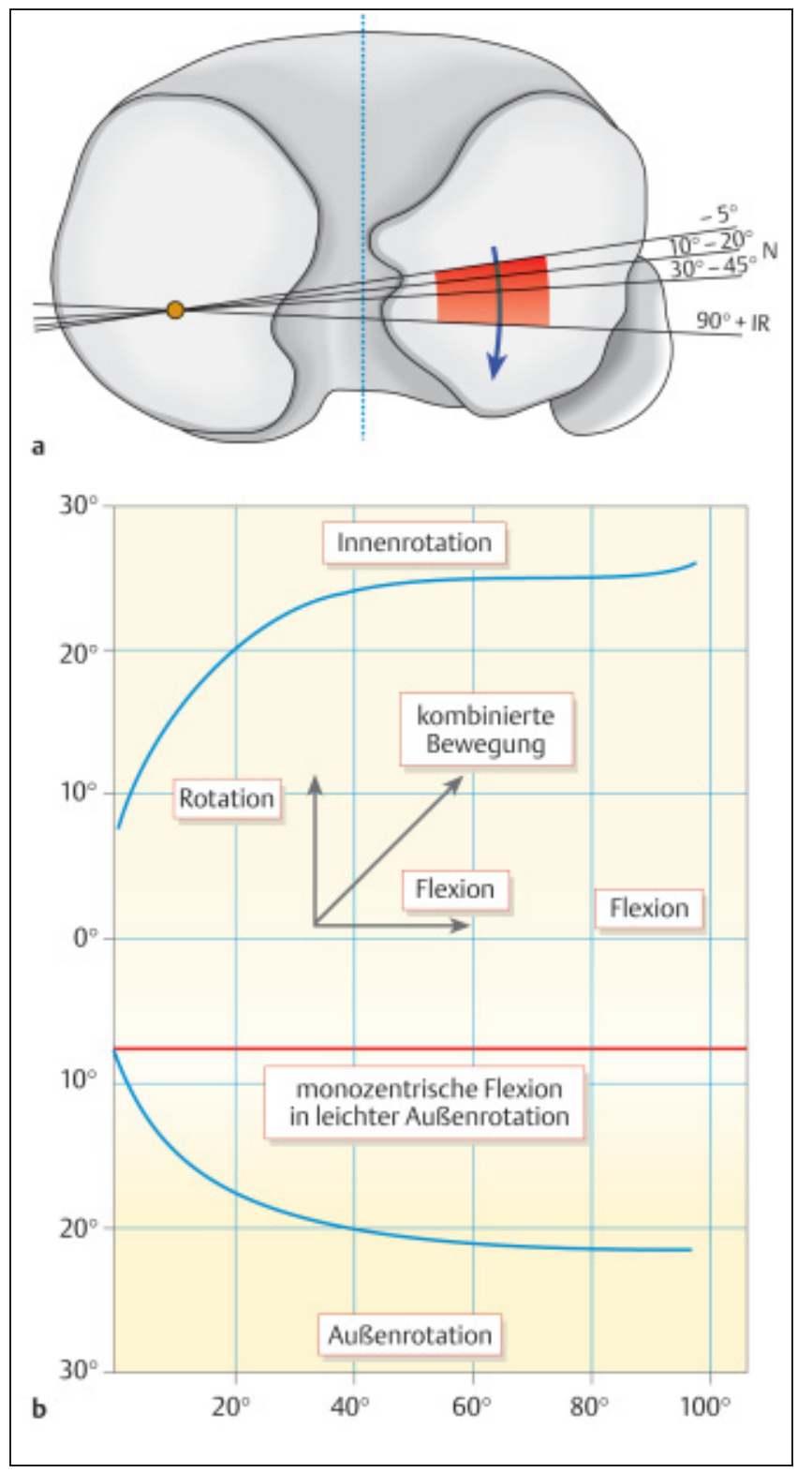

Abb. 7 Drehzentrum Abb. aus Literaturquelle 3 Hassenpflug H. Gekoppelte Knieendoprothese
Streckbewegung (Abb. 7). Durch die verringerte Bewegungsfreiheit entstehen jedoch Zwangskräfte, die durch Langschäfte kompensiert werden müssen. Hierdurch erfolgt die Krafteinleitung über die Diaphyse von Femur und Tibia und nicht über die Kondylen des Femurs und des Tibiaplateaus. Die 10-Jahre-Überlebenswahrscheinlichkeit für die gekoppelte Prothese liegt zwischen der des Unicondylären Schlittens und der Bicondylären KTP. Sie wurde von Hassenpflug für die Blauth-Prothese mit 92\% Orthopäde 2003 [3] angegeben. Großer Nachteil der gekoppelten Prothesen sind der große Knochenverlust bei der Implantation und hierdurch die mangelnde Rückzugsmöglichkeit auf andere Revisionsprothesen z.B.: beim Infekt, so dass auch eine
Arthrodese des Knies technisch schwierig durchzuführen ist und eine Oberschenkelamputation drohen kann.

Prinzipiell ist zu sagen, dass eine vollständige Konformität zwischen femoralen und tibialen Komponenten nur bei monozentrischen Beuge- Streck-Bewegungen erreicht werden kann. Die vermehrte Freigabe von Freiheitsgraden bedeutet unabdingbar, eine verschlechterte Kongruenz zwischen den Gelenkkomponenten $\mathrm{zu}$ akzeptieren und damit einen vermehrten Abrieb des Polyethylens der Tibiakomponente. Das heißt, dass eine größere Bewegungsfreiheit und damit ein physiologischer Bewegungsablauf immer mit einem erhöhten Verschleiß der Tibiakomponente erkauft wird, die zwar durch ein Mobile Bearing Plateau verbessert werden kann, jedoch nie die Kongruenz und damit den geringen Abriebverlust erreichen kann wie eine monozentrische Prothese.

Die Wahl der passenden Prothese richtet sich also streng nach dem vorliegenden Befund. Im Gegensatz zur primären sind bei der posttraumatischen Gonarthrose oftmals größere Achsfehlstellungen zu korrigieren. Dies erschwert deutlich ein befriedigendes Ligament Balancing $[9,10]$. Achsfehlstellungen des Femur von $>20^{\circ}$ und $>30^{\circ}$ der Tibia erlauben kein befriedigendes Soft Tissue Balancing [8], so dass hier ein Kniegelenksersatz nur in Kombination mit einer Umstellungsosteotomie erfolgen kann. Da meist mindest eine Osteosynthese vorausgegangen ist, findet sich in der Regel kein intakter Weichteilmantel mehr. Die Revisionsraten steigen aufgrund von Weichteilproblemen und Infekten auf bis zu $27 \%$ [2] und sind damit deutlich höher als bei der primären Gonarthrose $10 \%$.

Was Sir Charnley schon beim Kunstgelenkersatz an der Hüfte postulierte, gilt auch für die Knieprothese: „Die Implantation eines Kunstgelenks ist nicht das Ende, sondern der Anfang einer Krankheit". Man sollte daher vor allem bei dem meist jüngeren traumatologischen Patienten versuchen, den Kniegelenksersatz soweit wie möglich ins fortgeschrittene Alter zu verschieben.

Voraussetzung für eine erfolgreiche endoprothetische Versorgung bleibt weiterhin die suffizient durchgeführte Osteosynthese, die versucht, eine anatomische Rekonstruktion mit physiologischen Achs- und Bandverhältnissen zu schaffen. $[9,10]$.

Als nächster Schritt in der Behandlung der posttraumatischen Gonarthrose steht die Umstellungsosteotomie, sofern es sich nicht um eine Pangonarthrose handelt. Der Unicondyläre Schlitten stellt zumindest beim jungen Menschen keine adäquate Alternative dar und sollte dem Patienten zwischen 50 und 60 vorbehalten sein, um die Zeit bis zum Bicondylären Kniegelenksersatz zu überbrücken.

Die gekoppelte Prothese sollte wegen ihrer mangelnden Rückzugsmöglichkeiten durch den großen Knochenverlust dem Revisionseingriff vorbehalten bleiben, soweit es die Bandverhältnisse und die stabilisierende Muskelfunktion erlauben. 
Wenn alle endoprothetischen Möglichkeiten ausgeschöpft sind, bleibt letztendlich doch nur die Arthrodese des Kniegelenks oder sogar die Oberschenkelamputation. Dies muss insbesondere dem jungen Patienten bewusst gemacht werden, der voraussichtlich im Lauf seines Lebens mit mehreren Wechseloperationen $\mathrm{zu}$ rechnen hat.

\section{Zusammenfassung}

Die Knietotalendoprothese bei der posttraumatischen Gonarthrose ist trotz aller Probleme eine probate Möglichkeit, um eine befriedigende Funktion und Beschwerdearmut zu erreichen. Sie hat im Vergleich zur Arthrodese eine höhere Lebensqualität. Preis hierfür sind jedoch eine deutlich höhere postoperative Komplikationsrate sowie ein schlechteres funktionelles Ergebnis im Vergleich zu KTPs bei primärer Gonarthrose. Die Zukunft wird zeigen, ob durch Einführung der computerassistierten Chirurgie die Standzeiten der Knietotalendoprothesen verbessert werden können.

\section{Literatur}

${ }^{1}$ Buechel F, Knee F. Arthroplasty in Post-Traumatic Arthritits. In: The Journal of Arthroplasty. (Suppl. 1) 2002; 17: 63-68

${ }^{2}$ Gerich T, Bosch U, Schmidt E, Lobenhoffer P, Krettek C. Kniegelenkendoprothetik nach Tibiakopffrakturen. Der Unfallchirurg 2001; 5: 414-419

${ }^{3}$ Hassenpflug H. Gekoppelte Knieendoprothesen In: Der Orthopäde 2003; 32: $484-$ 489

${ }^{4}$ Khaled J, Saleh et al. Total Knee Arthroplasty after open Reduction and internal Fixation of Fractures of the Tibial Plateau. The Journal of Bone and Joint Surgery 2001; 1: 11441148

${ }^{5}$ König A, Kirschner S. Langzeitergebnisse in der Knieendoprothetik. Der Orthopäde 2003; 32: 516-526

${ }^{6}$ Labek G, Böhler N. Der minimal-invasive mediale Halbschlitten. Der Orthopäde 2003; 6: 454-460

${ }^{7}$ Nobuyuki Yoshino et al. Primary Total Knee Arthroplasty for Supracondylar/Condylar Femoral Fracture in Osteoarthritic Knees. In: The Journal of Arthroplasty 4 2001; 16 :

${ }^{8}$ Wang J-W, Wang C-J. Total Knee Arthroplasty for Arthritis of the Knee with Extra-Articular Deformity. The journal of bone and joint surgery 2002; 84: 1769-1774
${ }^{9}$ Weiss NG et al. Total Knee Arthroplasty in Post-Taumatic Arthrosis of the Knee. In: The Journal of Arthroplasty. 18 2003; 18 : $23-26$

${ }^{10}$ Weiss NG et al. Total Knee Arthroplasty in Patients with a Prior Fracture of the Tibial Plateau. In: The Journal of Bone and Joint Surgery 2003; 85: $218-221$

\section{Dr. med. Dietrich Schulte-Bockholt Oberarzt}

Dr. med. Klaus Wendl

Assistenzarzt

Dr. med. Alfred Schmidgen

Oberarzt

Prof. Dr. med. Andreas Wentzensen Ärztlicher Direktor

Klinik für Unfall- und Wiederherstellungschirurgie BG-Unfallklinik Ludwigshafen Unfallchirurgische Klinik an der Universität Heidelberg, Ludwig-Guttmann-Straße 13 D-67071 Ludwigshafen 